\title{
Mimetic Properties of Difference Operators: Product and Chain Rules as for Functions of Bounded Variation and Entropy Stability of Second Derivatives
}

\author{
Hendrik Ranocha \\ 7th September 2018
}

\begin{abstract}
For discretisations of hyperbolic conservation laws, mimicking properties of operators or solutions at the continuous (differential equation) level discretely has resulted in several successful methods. While well-posedness for nonlinear systems in several space dimensions is an open problem, mimetic properties such as summation-by-parts as discrete analogue of integration-by-parts allow a direct transfer of some results and their proofs, e.g. stability for linear systems.

In this article, discrete analogues of the generalised product and chain rules that apply to functions of bounded variation are considered. It is shown that such analogues hold for certain second order operators but are not possible for higher order approximations. Furthermore, entropy dissipation by second derivatives with varying coefficients is investigated, showing again the far stronger mimetic properties of second order approximations compared to higher order ones.
\end{abstract}

\section{Introduction}

Ever since the widespread application of computers in numerical mathematics and even before, finite difference methods have been successfully applied to differential equations. An important task is the development and investigation of stable and well-behaved numerical methods. While some general purpose methods can give satisfying results under certain circumstances, schemes that have been developed specifically for the target equation can be advantageous, e.g. if some properties of operators or solutions at the continuous level are mimicked discretely. This has been the goal of, e.g., geometric numerical integration methods for ordinary differential equations, cf. [16, 17].

In this regard, a well-developed theory of the problem at the continuous (differential equation) level is very important since it can be used as a guideline for the development of (semi-) discretisations. For linear systems of hyperbolic conservation laws, energy estimates play a fundamental role in the analysis of well-posedness [15]. An important technique is integrationby-parts. Thus, summation-by-parts (SBP) as a discrete analogue has been very successful, since manipulations at the continuous level can be mimicked discretely, yielding stability and 
conservation results, cf. $[2,3,21,40]$. Further references and results can be found in the review articles $[9,42]$.

Considering nonlinear equations such as scalar conservation laws, functions of locally bounded variation play an important role. Solutions of quasilinear equations can become discontinuous in finite time, even if smooth initial data and coefficients are given [5]. In his seminal work [45], Vol'pert investigated functions of locally bounded variation and their application to conservation laws. Since such functions can be discontinuous, he developed a corresponding notion of derivatives as measures. Moreover, he investigated products and compositions of functions of locally bounded variation and developed corresponding product and chain rules. Furthermore, the concept of bounded variation is important for the analysis of numerical methods for conservation laws since it implies compactness properties (Helly's theorem), cf. [19].

The investigation of semidiscretisations satisfying a single entropy inequality has received much interest, cf. $[10,11,13,14,24,30,32,33,39,43,44,48]$. For some conservation laws such as Burgers' equations, conservative corrections to the product rule can be used to obtain $L^{2}$ dissipative schemes, cf. $[12,35,36]$. Therefore, it is interesting whether the chain and product rules for functions of bounded variation have discrete analogues.

Furthermore, the investigation of numerical dissipation operators has received much interest, cf. [27, 34, 41, 47]. Such operators can be motivated by the vanishing viscosity approach to conservation laws, cf. [1]. For general entropies, the investigation of dissipation induced by such terms relies on the chain rule, cf. [23, Proof of Theorem I.3.4]. Thus, it is natural to investigate the entropy dissipation of difference approximations.

This article is structured as follows. At first, functions of bounded variation are briefly reviewed in section 2, focusing on the chain and product rules. Next, corresponding difference operators are investigated in section 3. It is proven that there are analogous product and chain rules for classical second order periodic and SBP operators (Lemma 3.1 and Lemma 3.2). Furthermore, it is proven that such analogues do not exist for higher order difference approximations of the first derivative (Theorem 3.6 and Theorem 3.8). Thereafter, dissipation operators approximating second derivatives with possibly varying coefficients are investigated in section 4. It is proven that certain second order difference operators are dissipative for every convex entropy (Theorem 4.1). Moreover, it is shown that such a result is impossible for discrete derivative operators with higher order of accuracy (Theorem 4.5). Finally, a summary and discussion is given in section 5 .

\section{Functions of Bounded Variation}

Functions of locally bounded variation, i.e. those locally integrable functions whose distributional first derivatives are Radon measures, play an important role in analysis, for example in the theory of scalar conservation laws as described in the seminal work of Vol'pert [45]. Further results about conservation laws and references can be found in the monograph [5], e.g. Theorem 6.2.6 and chapter XI. Some general results about functions of bounded variation can be found in $[8,46]$.

For functions of locally bounded variation, a product of a possibly discontinuous function and a measure occurs in both the chain rule and the product rule. If the function is integrable with respect to the measure, this product is well-defined as a measure, cf. [45]. In one space dimension, a function of bounded variation is continuous almost everywhere and the limits from the left and the right exist everywhere. If $u \in \mathrm{BV}_{\text {loc }}\left([a, b] ; \mathbb{R}^{m}\right)$ and $g: \mathbb{R}^{m} \rightarrow \mathbb{R}$ is (for simplicity) continuous, then Vol'pert [45] defined the averaged composition of $g$ and $u$ via

$$
\widehat{g(u)}(x):=\int_{0}^{1} g\left(u_{-}+s\left(u_{+}-u_{-}\right)\right) \mathrm{d} s,
$$

where $u_{ \pm}=\lim _{\varepsilon \searrow 0} u(x \pm \varepsilon)$ are the unique limits of $u$ from the left and right hand side, respectively. With this definition, the following chain and product rules have been obtained in $[45$, Section 13]. 
Theorem 2.1. If $u \in \operatorname{BV}\left([a, b] ; \mathbb{R}^{m}\right)$ and $f \in C^{1}\left(\mathbb{R}^{m} ; \mathbb{R}\right)$, the averaged composition $\overline{\partial_{u_{k}} f(u)}$ is locally integrable with respect to the measure $\partial_{x} u_{k}$ for $k \in\{1, \ldots, m\}, f(u) \in \mathrm{BV}_{\mathrm{loc}}$, and

$$
\partial_{x} f(u)=\sum_{k=1}^{m} \overline{\partial_{u_{k}} f(u)} \partial_{x} u_{k}
$$

In particular, for $\mathfrak{u}, \mathfrak{v} \in \mathrm{BV}[a, b]$,

$$
\partial_{x}(\mathfrak{u} \mathfrak{v})=\widehat{\mathfrak{u}} \partial_{x} \mathfrak{v}+\widehat{\mathfrak{v}} \partial_{x} \mathfrak{u} .
$$

Remark 2.2. Sometimes, it might be useful to distinguish vector valued functions $u \in \operatorname{BV}\left([a, b] ; \mathbb{R}^{m}\right)$, $m \geq 2$, and scalar valued functions $\mathfrak{u} \in \mathrm{BV}\left([a, b] ; \mathbb{R}^{1}\right)$ explicitly. In this case, a fracture font will be used for scalar valued functions. Nevertheless, the case $m=1$ is not excluded for vector valued functions $u \in \mathrm{BV}\left([a, b] ; \mathbb{R}^{m}\right)$ if not stated otherwise. If it is clear from the context whether a function is scalar valued or may be vector valued, the usual font will be used for simplicity.

Remark 2.3. If $\mathfrak{u}, \mathfrak{v} \in \mathrm{BV}[a, b]$, define $u \in \mathrm{BV}\left([a, b] ; \mathbb{R}^{2}\right)$ by $u(x)=(\mathfrak{u}(x), \mathfrak{v}(x))$. Considering the function $f \in C^{1}\left(\mathbb{R}^{2} ; \mathbb{R}\right)$, given by $f(u)=f\left(u_{1}, u_{2}\right)=u_{1} u_{2}$, the chain rule (2) becomes

$$
\partial_{x}(\mathfrak{u v})=\partial_{x} f(u)=\sum_{k=1}^{2} \overline{\partial_{u_{k}} f(u)} \partial_{x} u_{k}=\widehat{\mathfrak{v}} \partial_{x} \mathfrak{u}+\widehat{\mathfrak{u}} \partial_{x} \mathfrak{v} .
$$

Thus, the product rule (3) is indeed a special case of the chain rule (2).

The product rule (3) is also proven in the monograph [46, Section 6.4]. A generalisation of the corresponding definition of a possibly nonconservative product $f(u) \partial_{x} v$ has been developed and investigated by Dal Maso, LeFloch, and Murat [6]. See also [25, 37] for further studies.

In order to illustrate the general theory described above and lay some foundations for the following comparison with discrete derivative operators, two examples using jump functions will be considered. If $u, v \in \mathrm{BV}[a, b]$ are (scalar valued) jump functions,

$$
u(x)=\left\{\begin{array}{ll}
u_{-}, & x<0, \\
u_{+}, & x>0,
\end{array} \quad v(x)= \begin{cases}v_{-}, & x<0, \\
v_{+}, & x>0\end{cases}\right.
$$

they are of bounded variation and their derivatives are Radon measures. In particular, the derivatives $\partial_{x} u$ and $\partial_{x} v$ are multiples of the Dirac measure centred at zero. Viewing such a measure as a function mapping (measurable) sets to real numbers,

$$
\left(\partial_{x} u\right)(A)= \begin{cases}u_{+}-u_{-}, & \text {if } 0 \in A \\ 0, & \text { else. }\end{cases}
$$

Thus, the product rule (3) becomes in this case

$$
\begin{aligned}
\left(\partial_{x}(u v)\right)(\{0\}) & =u_{+} v_{+}-u_{-} v_{-} \\
& =\frac{u_{+}+u_{-}}{2}\left(v_{+}-v_{-}\right)+\frac{v_{+}+v_{-}}{2}\left(u_{+}-u_{-}\right) \\
& =\left(\widehat{u} \partial_{x} v\right)(\{0\})+\left(\widehat{v} \partial_{x} u\right)(\{0\}),
\end{aligned}
$$

where the measures on both sides of (3) have been applied to the set $\{0\}$ containing only the jump point. Similarly, for $f \in C^{1}$, the chain rule (2) becomes

$$
\begin{aligned}
\left(\partial_{x} f(u)\right)(\{0\})=f\left(u_{+}\right)-f\left(u_{-}\right)=\int_{0}^{1} f^{\prime}\left(u_{-}+s\left(u_{+}-u_{-}\right)\right) \mathrm{d} s \cdot\left(u_{+}-u_{-}\right) & =\left(\widehat{f^{\prime}(u)} \cdot \partial_{x} u\right)(\{0\}) .
\end{aligned}
$$

Of course, the intermediate steps of (7) and (8) can also be seen as proofs of the general product and chain rule in this special case.

Interpreting the difference $u_{+}-u_{-}$as a discrete derivative, (7) is a discrete product rule and (8) is a discrete chain rule. Both use averages instead of the usual point values occurring in the continuous analogues for differentiable functions. Thus, it is interesting whether this can be generalised. 


\section{Difference Operators}

Consider a general discrete derivative/difference operator $D$, acting on grid functions $\mathbf{u}=$ $\left(\mathbf{u}_{i}\right)_{i}=\left(u\left(x_{i}\right)\right)_{i}$ defined on a possibly non-uniform grid with nodes $x_{i} \in \mathbb{R}$ and $h:=\min _{i}\left(x_{i+1}-\right.$ $\left.x_{i}\right)>0$. Note that this includes both classical finite difference operators and spectral collocation operators such as nodal discontinuous Galerkin ones.

In practice, the grid function is represented by the vector of its point values and the discrete derivative operator by a matrix with entries $D_{i j}$. General nonlinear operations such as composition or multiplication are conducted pointwise, i.e. if $\mathbf{u}$ and $\mathbf{v}$ are two grid functions, their product $\mathbf{u v}$ is the grid function with components $(\mathbf{u v})_{i}=\mathbf{u}_{i} \mathbf{v}_{i}$.

\subsection{Classical Second Order Derivative Operators}

The classical second order finite difference operator on a uniform grid is given by

$$
(D \mathbf{u})_{i}=\frac{\mathbf{u}_{i+1}-\mathbf{u}_{i-1}}{2 h} \approx u^{\prime}\left(x_{i}\right) .
$$

The corresponding summation-by-parts (SBP) operator uses this stencil in the interior and if the nodes $x_{0}, \ldots, x_{N}$ are used - the boundary closures

$$
(D \mathbf{u})_{0}=\frac{\mathbf{u}_{1}-\mathbf{u}_{0}}{h} \approx u^{\prime}\left(x_{0}\right), \quad(D \mathbf{u})_{N}=\frac{\mathbf{u}_{N}-\mathbf{u}_{N-1}}{h} \approx u^{\prime}\left(x_{N}\right) .
$$

Analogously to the product rule (7) for a step function of bounded variation, considering scalar valued grid functions $\mathbf{u}$ and $\mathbf{v}$,

$$
\begin{aligned}
(D \mathbf{u v})_{i} & =\frac{\mathbf{u}_{i+1} \mathbf{v}_{i+1}-\mathbf{u}_{i-1} \mathbf{v}_{i-1}}{2 h} \\
& =\frac{\mathbf{u}_{i+1}+\mathbf{u}_{i-1}}{2} \frac{\mathbf{v}_{i+1}-\mathbf{v}_{i-1}}{2 h}+\frac{\mathbf{u}_{i+1}-\mathbf{u}_{i-1}}{2 h} \frac{\mathbf{v}_{i+1}+\mathbf{v}_{i-1}}{2} \\
& =(A \mathbf{u})_{i}(D \mathbf{v})_{i}+(D \mathbf{u})_{i}(A \mathbf{v})_{i},
\end{aligned}
$$

if the averaging operator $A$ is defined by

$$
(A \mathbf{u})_{i}=\frac{\mathbf{u}_{i+1}+\mathbf{u}_{i-1}}{2} \approx u\left(x_{i}\right) .
$$

For the corresponding SBP operator, the terms at the left boundary are

$$
\begin{aligned}
(D \mathbf{u v})_{0} & =\frac{\mathbf{u}_{1} \mathbf{v}_{1}-\mathbf{u}_{0} \mathbf{v}_{0}}{h} \\
& =\frac{\mathbf{u}_{1}+\mathbf{u}_{0}}{2} \frac{\mathbf{v}_{1}-\mathbf{v}_{0}}{h}+\frac{\mathbf{u}_{1}-\mathbf{u}_{0}}{h} \frac{\mathbf{v}_{1}+\mathbf{v}_{0}}{2}=(A \mathbf{u})_{0}(D \mathbf{v})_{0}+(D \mathbf{u})_{0}(A \mathbf{v})_{0} ，
\end{aligned}
$$

if the boundary closures of $A$ are given by

$$
(A \mathbf{u})_{0}=\frac{\mathbf{u}_{1}+\mathbf{u}_{0}}{2} \approx u\left(x_{0}\right), \quad(A \mathbf{u})_{N}=\frac{\mathbf{u}_{N}+\mathbf{u}_{N-1}}{2} \approx u\left(x_{N}\right) .
$$

The terms at the right boundary are similar. This is summed up in

Lemma 3.1. The classical second order derivative operator $D$ (on a periodic grid or with boundary closures given above) fulfils the product rule

$$
D(\mathbf{u v})=(A \mathbf{u})(D \mathbf{v})+(D \mathbf{u})(A \mathbf{v}),
$$

where the averaging operator $A$ defined above is of the same order of accuracy as the derivative operator $D$, i.e. it fulfils $(A \mathbf{u})_{i}=u\left(x_{i}\right)+O\left(h^{2}\right)$ in the interior and $(A \mathbf{u})_{0, N}=u\left(x_{0, N}\right)+O(h)$ at the boundaries for a smooth function $u$. 
Similarly, a general chain rule as discrete analogue of (8) is satisfied. Indeed, if $f$ is continuously differentiable and $\mathbf{u}$ a possibly vector valued grid function,

$$
\begin{aligned}
(D f(\mathbf{u}))_{i} & =\frac{f\left(\mathbf{u}_{i+1}\right)-f\left(\mathbf{u}_{i-1}\right)}{2 h} \\
& =\underbrace{\int_{0}^{1} f^{\prime}\left(\mathbf{u}_{i-1}+s\left(\mathbf{u}_{i+1}-\mathbf{u}_{i-1}\right)\right) \mathrm{d} s}_{=:\left(A_{f^{\prime}} \mathbf{u}\right)_{i}} \cdot \frac{\mathbf{u}_{i+1}-\mathbf{u}_{i-1}}{2 h}=\left(A_{f} \mathbf{u}\right)_{i} \cdot(D \mathbf{u})_{i}
\end{aligned}
$$

for interior nodes, where the possibly nonlinear averaging operator $A_{f^{\prime}}$ has been introduced. At the boundary nodes, it is given by

$$
\begin{aligned}
\left(A_{f^{\prime}} \mathbf{u}\right)_{0} & =\int_{0}^{1} f^{\prime}\left(\mathbf{u}_{0}+s\left(\mathbf{u}_{1}-\mathbf{u}_{0}\right)\right) \mathrm{d} s \approx f^{\prime}\left(u\left(x_{0}\right)\right) \\
\left(A_{f^{\prime}} \mathbf{u}\right)_{N} & =\int_{0}^{1} f^{\prime}\left(\mathbf{u}_{N-1}+s\left(\mathbf{u}_{N}-\mathbf{u}_{N-1}\right)\right) \mathrm{d} s \approx f^{\prime}\left(u\left(x_{N}\right)\right) .
\end{aligned}
$$

This is summed up in

Lemma 3.2. The classical second order derivative operator D (on a periodic grid or with boundary closures) satisfies the chain rule

$$
D f(\mathbf{u})=\left(A_{f} \mathbf{u}\right) \cdot(D \mathbf{u}),
$$

where the averaging operator $A_{f^{\prime}}$ defined above is of the same order of accuracy as the derivative operator $D$, i.e. it fulfils $\left(A_{f^{\prime}} \mathbf{u}\right)_{i}=f^{\prime}\left(u\left(x_{i}\right)\right)+O\left(h^{2}\right)$ in the interior and $\left(A_{f^{\prime}} \mathbf{u}\right)_{0, N}=f^{\prime}\left(u\left(x_{0, N}\right)\right)+O(h)$ at the boundaries for smooth (and possibly vector valued) functions $u$ and $f$.

Remark 3.3. The averaging operator $A$ used for the product rule is a special case of the general averaging operator $A_{f^{\prime}}$. Indeed, $A=A_{\mathrm{id}}$, where id is the identity mapping.

Remark 3.4. In general, $A_{f}$ is neither a linear operator nor an averaging operator acting on $f^{\prime}(\mathbf{u})$. Instead, it is a possibly nonlinear operator that uses intermediate values of $\mathbf{u}$ to average $f^{\prime}$. It is linear if and only if $f^{\prime}$ is linear, in particular in the case $f^{\prime}=$ id, i.e. $A_{\text {id }}=A$ discussed in Remark 3.3.

\subsection{Higher Order Derivative Operators}

The product and chain rules for second order derivative operators cannot be generalised to higher order derivative operators. In order to prove this, the asymptotic expansion of the error of the derivative operator will be used.

Lemma 3.5. Assume that $D$ is a discrete derivative operator of order $p$, i.e. $(D \mathbf{u})_{i}=u^{\prime}\left(x_{i}\right)+O\left(h^{p}\right)$ or, equivalently, $D$ is exact for polynomials of degree $\leq p$, with $p$ maximal. If $u$ is a smooth scalar-valued function,

$$
(D \mathbf{u})_{i}=u^{\prime}\left(x_{i}\right)+u^{(p+1)}\left(x_{i}\right) C_{i}^{D} h^{p}+O\left(h^{p+1}\right),
$$

where $C_{i}^{D} h^{p}=O\left(h^{p}\right)$ depends only on the grid and the derivative operator.

Proof. By Taylor expansion, using the exactness of $D$ for polynomials of degree $\leq p$,

$$
\begin{aligned}
& (D \mathbf{u})_{i}=\sum_{j} D_{i j} \mathbf{u}_{j}=\sum_{j} D_{i j} u\left(x_{j}\right) \\
= & \sum_{j} D_{i j}\left(u\left(x_{i}\right)+u^{\prime}\left(x_{i}\right)\left(x_{j}-x_{i}\right)+\cdots+\frac{1}{(p+1) !} u^{(p+1)}\left(x_{i}\right)\left(x_{j}-x_{i}\right)^{p+1}+O\left(h^{p+2}\right)\right) \\
= & u^{\prime}\left(x_{i}\right)+u^{(p+1)}\left(x_{i}\right) \underbrace{\sum_{j} \frac{1}{(p+1) !} D_{i j}\left(x_{j}-x_{i}\right)^{p+1}}_{=: C_{i}^{D} h^{p}}+O\left(h^{p+1}\right) .
\end{aligned}
$$

Here, $C_{i}^{D} h^{p}=O\left(h^{p}\right)$, since $D$ scales as $h^{-1}$. 
This can be used to prove one of the main observations of this article.

Theorem 3.6. If $D$ is a discrete derivative operator of order $p>2$, there can be no averaging operator $A$ of order $q \in \mathbb{N}$ such that there is a product rule of the form $D(\mathbf{u v})=(A \mathbf{u})(D \mathbf{v})+(D \mathbf{u})(A \mathbf{v})$.

Proof. Consider the asymptotic expansions

$$
(D(\mathbf{u v}))_{i}=(u v)^{\prime}\left(x_{i}\right)+(u v)^{(p+1)}\left(x_{i}\right) C_{i}^{D} h^{p}+O\left(h^{p+1}\right)
$$

and

$$
\begin{aligned}
& (A \mathbf{u})_{i}(D \mathbf{v})_{i}=\left(u\left(x_{i}\right)+C_{i}^{A}(u) h^{q}+O\left(h^{q+1}\right)\right)\left(v^{\prime}\left(x_{i}\right)+v^{(p+1)}\left(x_{i}\right) C_{i}^{D} h^{p}+O\left(h^{p+1}\right)\right), \\
& (D \mathbf{u})_{i}(A \mathbf{v})_{i}=\left(u^{\prime}\left(x_{i}\right)+u^{(p+1)}\left(x_{i}\right) C_{i}^{D} h^{p}+O\left(h^{p+1}\right)\right)\left(v\left(x_{i}\right)+C_{i}^{A}(v) h^{q}+O\left(h^{q+1}\right)\right),
\end{aligned}
$$

where $C_{i}^{A}(u)$ is the leading order coefficient for $A$ and may depend on the function $u$ and its derivatives. There are three different cases: $q<p, q=p$, and $q>p$.

If $q<p$, the product rule cannot hold, because the terms

$$
\left(u^{\prime}\left(x_{i}\right) C_{i}^{A}(v)+v^{\prime}\left(x_{i}\right) C_{i}^{A}(u)\right) h^{q}
$$

involving $h^{q}$ are not matched by terms in (21). Basically, $D(\mathbf{u v})$ is a $p$-th order approximation while $(A \mathbf{u})(D \mathbf{v})+(D \mathbf{u})(A \mathbf{v})$ is only a $q$-th order approximation.

If $q=p$, the product rule can only hold if the terms involving $h^{p}=h^{q}$ are equal, i.e. if

$$
\begin{aligned}
& (u v)^{(p+1)}\left(x_{i}\right) C_{i}^{D} \\
& =\left(u\left(x_{i}\right) v^{(p+1)}\left(x_{i}\right)+u^{(p+1)}\left(x_{i}\right) v\left(x_{i}\right)\right) C_{i}^{D}+u^{\prime}\left(x_{i}\right) C_{i}^{A}(v)+v^{\prime}\left(x_{i}\right) C_{i}^{A}(u) .
\end{aligned}
$$

Since

$$
(u v)^{(p+1)}\left(x_{i}\right)=\sum_{k=0}^{p+1}\left(\begin{array}{c}
p+1 \\
k
\end{array}\right) u^{(k)}\left(x_{i}\right) v^{(p+1-k)}\left(x_{i}\right),
$$

the terms with $k=0$ and $k=p+1$ match the braces on the right hand side of (24), but the remaining terms can only match if $p \leq 2$, since the remaining sum cannot be factored as on the right hand side.

Finally, if $q>p$, the terms involving $h^{p}$ do not match, because

$$
(u v)^{(p+1)}\left(x_{i}\right) \neq u\left(x_{i}\right) v^{(p+1)}\left(x_{i}\right)+u^{(p+1)}\left(x_{i}\right) v\left(x_{i}\right)
$$

for $p \geq 1$ in general.

Remark 3.7. Using polynomial collocation methods on Lobatto Legendre or Gauss Legendre nodes in $[-1,1]$, a discrete product rule holds for $p=1$, i.e. for two nodes, since they are of the same form as the classical finite difference derivative operator. However, for $p=2$, there can be no product rule. Indeed, for Lobatto nodes $\{-1,0,1\}$ and $u(x)=(1+x)^{2}=v(x)$, the discrete derivatives of $u$ and $v$ at -1 are zero (since they are exact), but the discrete derivative of $u v$ at -1 is

$$
(D \mathbf{u v})_{-1}=-\frac{3}{2} \mathbf{u}_{-1} \mathbf{v}_{-1}+2 \mathbf{u}_{0} \mathbf{v}_{0}-\frac{1}{2} \mathbf{u}_{1} \mathbf{v}_{1}=0+2 \cdot 1^{2}-\frac{1}{2} \cdot 4^{2}=-6 \neq 0 .
$$

A similar argument holds for Gauss Legendre nodes.

Since the product rule is a special case of the chain rule with vector valued functions $u$ (cf. Remark 2.3), a general chain rule is also excluded for discrete derivative operators of higher order of accuracy. However, this argument does not forbid a chain rule for scalar valued functions. Nevertheless, this case is also excluded by the second main observation of this article.

Theorem 3.8. If $D$ is a discrete derivative operator of order $p>2$, there can be no general averaging operator $A_{f^{\prime}}$ of order $q \in \mathbb{N}$ such that there is a chain rule of the form $D(f(\mathbf{u}))=\left(A_{f^{\prime}} \mathbf{u}\right) \cdot(D \mathbf{u})$. 
Proof. By the argument above, it suffices to consider scalar valued functions. In this case,

$$
(D f(\mathbf{u}))_{i}=f^{\prime}\left(\mathbf{u}_{i}\right) u^{\prime}\left(x_{i}\right)+(f(u))^{(p+1)}\left(x_{i}\right) C_{i}^{D} h^{p}+O\left(h^{p+1}\right)
$$

and

$$
\begin{aligned}
\left(A_{f^{\prime}} \mathbf{u}\right)_{i}(D \mathbf{u})_{i}=\left(f^{\prime}\left(\mathbf{u}_{i}\right)+C_{i}^{A}\left(f^{\prime}(u)\right) h^{q}+O\left(h^{q+1}\right)\right) & \\
& \left(u^{\prime}\left(x_{i}\right)+u^{(p+1)}\left(x_{i}\right) C_{i}^{D} h^{p}+O\left(h^{p+1}\right)\right) .
\end{aligned}
$$

Again, there are three different cases: $q<p, q=p$, and $q>p$.

If $q<p$, the chain rule cannot hold, because $D(f(\mathbf{u}))$ is a $p$-th order approximation while $\left(A_{f}, \mathbf{u}\right)(D u)$ is only a $q$-th order approximation.

If $q=p,(f(u))^{(p+1)}\left(x_{i}\right)$ can be expressed using the formula of Faà di Bruno [18, Lemma II.2.8, simplified for the scalar case],

$$
(f(u))^{(p+1)}\left(x_{i}\right)=\sum_{u \in L S_{p+2}} f^{(m)}\left(\mathbf{u}_{i}\right) u^{\left(\delta_{1}\right)}\left(x_{i}\right) \ldots u^{\left(\delta_{m}\right)}\left(x_{i}\right) .
$$

Here, $L S_{p+2}$ is the set of special labelled trees of order $p+2$ which have no ramifications except at the root, $m$ is the number of branches leaving the root, and $\delta_{1}, \ldots, \delta_{m}$ are the numbers of nodes in each of these branches, see [18, Lemma II.2.8]. Thus, it is clear that $u^{\prime}\left(x_{i}\right)$ cannot be factored out of the remaining terms after subtracting $f^{\prime}\left(\mathbf{u}_{i}\right) u^{(p+1)}\left(x_{i}\right)$ if $p>2$.

Finally, if $q>p$, the terms involving $h^{p}$ do not match, because

$$
(f(u))^{(p+1)}\left(x_{i}\right) \neq f^{\prime}\left(\mathbf{u}_{i}\right) u^{(p+1)}\left(x_{i}\right)
$$

for $p \geq 1$ in general.

Remark 3.9. A product rule for classical difference operators with error term of the form

$$
(D \mathbf{u v})_{i}=\mathbf{u}_{i}(D \mathbf{v})_{i}+\left(\partial_{x} u\right)_{i}(A \mathbf{v})_{i}+e_{i}
$$

has been used in [28, Lemma 3.1 and Lemma 3.2]. If $u$ is smooth, $\left(\partial_{x} u\right)_{i}$ is the derivative at $x_{i}$ and $\|e\| \leq C h\|\mathbf{v}\|$ for some constant $C>0$. The averaging operator $A$ is linear and of the same order of accuracy as the derivative operator $D$.

Remark 3.10. The investigation of discrete product and chain rules is also somewhat loosely related to the entropy stability and conservation theory initiated by Tadmor [43, 44]. Indeed, instead of a chain rule of the form $\partial_{x} f(u)=\overline{f^{\prime}(u)} \partial_{x} u(2)$, a discrete version of $U^{\prime}(u) \cdot \overline{\partial_{x} f(u)}=$ $\overline{\partial_{x} F(u)}$ is used, where $U$ is the entropy fulfilling $U^{\prime}(u) \cdot f^{\prime}(u)=F^{\prime}(u)$. Such approximations can be found for arbitrary order, cf. [4, 10, 24, 30,39]. Basically, schemes of lower order can be extrapolated if regular grids are used, cf. [31, Section 3.2]. Nevertheless, they can be used also on certain irregular grids.

\section{Entropy Stability of Discrete Second Derivatives}

In order to regularise a hyperbolic conservation law $\partial_{t} u+\partial_{x} f(u)=0$, where $u$ are the conserved variables and $f(u)$ is the flux, a parabolic term can be added to the right-hand side, resulting in

$$
\partial_{t} u(t, x)+\partial_{x} f(u(t, x))=\partial_{x}\left(\varepsilon(x) \partial_{x} u(t, x)\right),
$$

where $\varepsilon \geq 0$ controls the amount of viscosity. An entropy is a convex function $U$ satisfying $U^{\prime}(u) \cdot f^{\prime}(u)=F^{\prime}(u)$, where $F$ is the corresponding entropy flux. Thus, smooth solutions of the conservation law fulfil the additional conservation law

$$
\partial_{t} U(u)=U^{\prime}(u) \cdot \partial_{t} u=-U^{\prime}(u) \cdot f^{\prime}(u) \cdot \partial_{x} u=-\partial_{x} F(u)
$$


and an entropy inequality $\partial_{t} U+\partial_{x} F \leq 0$ is required for weak solutions, cf. [5, Chapter IV]. The viscosity term on the right-hand side induces a global entropy inequality for sufficiently smooth solutions. Indeed, in a periodic domain $\Omega$,

$$
\int_{\Omega} U^{\prime} \cdot \partial_{x}\left(\varepsilon \partial_{x} u\right) \mathrm{d} x=-\int_{\Omega} \varepsilon\left(\partial_{x} U^{\prime}\right) \cdot \partial_{x} u \mathrm{~d} x=-\int_{\Omega} \varepsilon\left(\partial_{x} u\right) \cdot U^{\prime \prime} \cdot \partial_{x} u \mathrm{~d} x \leq 0,
$$

since $U$ is convex and $\varepsilon \geq 0$. In a non-periodic domain $\Omega$, if $\varepsilon$ vanishes on $\partial \Omega$, the same result holds. Otherwise, there will be additional boundary terms.

The computation in (35) relies on the chain rule. Thus, it might be conjectured that second order difference approximations of the Laplace operator (with possibly varying coefficients) are also dissipative for every entropy $U$ and that higher order difference approximations to the second derivative are not necessarily dissipative for every entropy $U$.

\subsection{Second Order Derivative Operators}

In a periodic domain, the classical second order difference approximation to the Laplace operator is given by

$$
\left(D_{2} \mathbf{u}\right)_{i}=\frac{\mathbf{u}_{i+1}-2 \mathbf{u}_{i}+\mathbf{u}_{i-1}}{h^{2}} .
$$

Thus, multiplying pointwise by $U^{\prime}\left(\mathbf{u}_{i}\right)=U_{i}^{\prime}$ and summing up all terms yields due to the periodicity of the domain

$$
\begin{aligned}
h^{2} \sum_{i} U_{i}^{\prime} \cdot\left(D_{2} \mathbf{u}\right)_{i} & =\sum_{i} U_{i}^{\prime} \cdot\left(\mathbf{u}_{i+1}-\mathbf{u}_{i}\right)-\sum_{i} U_{i}^{\prime} \cdot\left(\mathbf{u}_{i}-\mathbf{u}_{i-1}\right) \\
& =-\sum_{i}\left(U_{i+1}^{\prime}-U_{i}^{\prime}\right) \cdot\left(\mathbf{u}_{i+1}-\mathbf{u}_{i}\right) \leq 0
\end{aligned}
$$

since $U^{\prime}$ is monotone. If $u$ is scalar valued, $U^{\prime}$ is monotonically increasing, since $U^{\prime \prime} \geq 0$ due to the convexity of $U$. If $u$ is vector valued, the usual generalised definition of monotonicity is used, i.e. $\left(U^{\prime}(u)-U^{\prime}(v)\right) \cdot(u-v) \geq 0$, cf. [38, section II.2, p. 37]. Indeed, due to the convexity of $U$,

$$
\begin{aligned}
& \left(\mathbf{u}_{i+1}-\mathbf{u}_{i}\right) \cdot\left(U^{\prime}\left(\mathbf{u}_{i+1}\right)-U^{\prime}\left(\mathbf{u}_{i}\right)\right) \\
& \quad=\int_{0}^{1}\left(\mathbf{u}_{i+1}-\mathbf{u}_{i}\right) \cdot U^{\prime \prime}\left(\mathbf{u}_{i}+s\left(\mathbf{u}_{i+1}-u_{i}\right)\right) \cdot\left(\mathbf{u}_{i+1}-\mathbf{u}_{i}\right) \mathrm{d} s \geq 0 .
\end{aligned}
$$

This is exactly the chain rule for classical difference approximations.

If a variable coefficient $\varepsilon \geq 0$ is considered in a periodic domain, a second order approximation to $\partial_{x}\left(\varepsilon \partial_{x} u\right)$ is given by

$$
\left(D_{2}^{\varepsilon} \mathbf{u}\right)_{i}=\frac{\varepsilon_{i}+\varepsilon_{i+1}}{2 h^{2}} \mathbf{u}_{i+1}-\frac{\varepsilon_{i-1}+2 \varepsilon_{i}+\varepsilon_{i+1}}{2 h^{2}} \mathbf{u}_{i}+\frac{\varepsilon_{i-1}+\varepsilon_{i}}{2 h^{2}} \mathbf{u}_{i-1},
$$

cf. [26]. Using again the periodicity and the convexity of $U$,

$$
\begin{aligned}
h^{2} \sum_{i} U_{i}^{\prime} \cdot\left(D_{2}^{\varepsilon} \mathbf{u}\right)_{i} & =\sum_{i} \frac{\varepsilon_{i}+\varepsilon_{i+1}}{2} U_{i}^{\prime} \cdot\left(\mathbf{u}_{i+1}-\mathbf{u}_{i}\right)-\sum_{i} \frac{\varepsilon_{i-1}+\varepsilon_{i}}{2} U_{i}^{\prime} \cdot\left(\mathbf{u}_{i}-\mathbf{u}_{i-1}\right) \\
& =-\sum_{i} \frac{\varepsilon_{i}+\varepsilon_{i+1}}{2}\left(U_{i+1}^{\prime}-U_{i}^{\prime}\right) \cdot\left(\mathbf{u}_{i+1}-\mathbf{u}_{i}\right) \leq 0 .
\end{aligned}
$$

Summation-by-parts operators for second derivatives with variable coefficients have been developed in [26]. The second order discrete derivative in the interior is given by (39) and equipped with the boundary closures

$$
\begin{aligned}
\left(D_{2}^{\varepsilon} \mathbf{u}\right)_{0} & =\left(2 \varepsilon_{0}-\varepsilon_{1}\right) \mathbf{u}_{0}+\left(-3 \varepsilon_{0}+\varepsilon_{1}\right) \mathbf{u}_{1}+\varepsilon_{0} \mathbf{u}_{3} \\
\left(D_{2}^{\varepsilon} \mathbf{u}\right)_{N} & =\left(2 \varepsilon_{N}-\varepsilon_{N-1}\right) \mathbf{u}_{N}+\left(-3 \varepsilon_{N}+\varepsilon_{N-1}\right) \mathbf{u}_{N-1}+\varepsilon_{N} \mathbf{u}_{N-2} .
\end{aligned}
$$


If the variable coefficient $\varepsilon$ vanishes at the boundary, i.e. if $\varepsilon_{0}=0=\varepsilon_{N}$, these boundary closures become

$$
\left(D_{2}^{\varepsilon} \mathbf{u}\right)_{0}=\varepsilon_{1}\left(\mathbf{u}_{1}-\mathbf{u}_{0}\right), \quad\left(D_{2}^{\varepsilon} \mathbf{u}\right)_{N}=-\varepsilon_{N-1}\left(\mathbf{u}_{N}-\mathbf{u}_{N-1}\right) .
$$

Since the discrete integral is given as a quadrature with weights on the diagonal of the mass/norm matrix $H=\operatorname{diag}(1 / 2,1, \ldots, 1,1 / 2)$, the discrete equivalent of the integral $\int_{\Omega} U^{\prime}$. $\partial_{x}\left(\varepsilon \partial_{x} u\right)$ is

$$
\begin{aligned}
& \sum_{i=0}^{N} H_{i i} U_{i}^{\prime} \cdot\left(D_{2}^{\varepsilon} \mathbf{u}\right)_{i} \\
= & \frac{1}{2} \varepsilon_{1} U_{0}^{\prime} \cdot\left(\mathbf{u}_{1}-\mathbf{u}_{0}\right)-\frac{1}{2} \varepsilon_{N-1} U_{N}^{\prime} \cdot\left(\mathbf{u}_{N}-\mathbf{u}_{N-1}\right) \\
& +\sum_{i=1}^{N-1} \frac{\varepsilon_{i}+\varepsilon_{i+1}}{2} U_{i}^{\prime} \cdot\left(\mathbf{u}_{i+1}-\mathbf{u}_{i}\right)-\sum_{i=1}^{N-1} \frac{\varepsilon_{i-1}+\varepsilon_{i}}{2} U_{i}^{\prime} \cdot\left(\mathbf{u}_{i}-\mathbf{u}_{i-1}\right) \\
= & \frac{\varepsilon_{0}+\varepsilon_{1}}{2} U_{0}^{\prime} \cdot\left(\mathbf{u}_{1}-\mathbf{u}_{0}\right)-\frac{\varepsilon_{N-1}+\varepsilon_{N}}{2} U_{N}^{\prime} \cdot\left(\mathbf{u}_{N}-\mathbf{u}_{N-1}\right) \\
& +\sum_{i=1}^{N-1} \frac{\varepsilon_{i}+\varepsilon_{i+1}}{2} U_{i}^{\prime} \cdot\left(\mathbf{u}_{i+1}-\mathbf{u}_{i}\right)-\sum_{i=0}^{N-2} \frac{\varepsilon_{i}+\varepsilon_{i+1}}{2} U_{i+1}^{\prime} \cdot\left(\mathbf{u}_{i+1}-\mathbf{u}_{i}\right) \\
= & -\sum_{i=0}^{N} \frac{\varepsilon_{i}+\varepsilon_{i+1}}{2}\left(U_{i+1}^{\prime}-U_{i}^{\prime}\right) \cdot\left(\mathbf{u}_{i+1}-\mathbf{u}_{i}\right) \\
\leq & 0 .
\end{aligned}
$$

This proves

Theorem 4.1. The discretisations $D_{2}^{\varepsilon}$ of the second derivative operator $\partial_{x}\left(\varepsilon \partial_{x} \cdot\right)$ with possibly varying coefficients $\varepsilon \geq 0$ given above in periodic domains or on bounded domains with $\varepsilon_{0}=0=\varepsilon_{N}$ are entropy dissipative for every convex entropy.

Remark 4.2. The statement of Theorem 4.1 holds for the second order summation-by-parts operator $D_{2}^{\varepsilon}$ (and its interior stencil in periodic domains) mentioned above. It is not necessarily true for every second order approximation of $\partial_{x}\left(\varepsilon \partial_{x} \cdot\right)$. Indeed, in a periodic domain, such an approximation is also given by

$$
\left(\widetilde{D}_{2}^{\varepsilon} \mathbf{u}\right)_{i}=\varepsilon_{i} \frac{\mathbf{u}_{i+1}-2 \mathbf{u}_{i}+\mathbf{u}_{i-1}}{h^{2}}+\frac{\varepsilon_{i+1}-\varepsilon_{i-1}}{2 h} \frac{\mathbf{u}_{i+1}-\mathbf{u}_{i-1}}{2 h} .
$$

Choose the grid $x_{i}=i, i \in\{0,1,2,3\}$, with periodic boundary conditions, i.e. $\mathbf{u}_{3}=\mathbf{u}_{0}$. Set $\mathbf{u}=\left(\mathbf{u}_{0}, \mathbf{u}_{1}, \mathbf{u}_{2}\right)=(0.6,0.8,0.2), \varepsilon=(0.4,0.2,0.8)$ and use the entropy given by $U(u)=u$. Then, $U^{\prime}(u)=1$ and

$$
\sum_{i=0}^{2} U^{\prime}\left(\mathbf{u}_{i}\right) \cdot\left(\widetilde{D}_{2}^{\varepsilon} \mathbf{u}\right)_{i}=-0.17-0.20+0.79=0.42>0 .
$$

While this does not prove that the SBP operator mentioned above is the only second order entropy dissipative approximation, it illustrates the good properties of this operator.

\subsection{Higher Order Derivative Operators}

Since there is no discrete chain rule for higher order difference approximations to the first derivative, it might be conjectured that discrete higher order second derivatives are in general not entropy dissipative. In order to prove this, it suffices to consider the case of constant coefficients. At the grid point $x_{j}$, a general (linear) discrete approximation of the second derivative can be written as

$$
\left(D_{2} \mathbf{u}\right)_{j}=\sum_{k} c_{k} \mathbf{u}_{j+k}
$$

The following result will be used. 
Lemma 4.3. If (46) is an approximation of the second derivative with order of accuracy $p>2$, there is a $k \neq 0$ such that $c_{k}<0$.

Proof. Using Taylor expansion, the order conditions for an order of accuracy $p=3$ are

$$
\begin{array}{rlrl}
\sum_{k} c_{k} & =0, \quad \sum_{k} c_{k}\left(x_{j+k}-x_{j}\right) & =0, & \sum_{k} c_{k}\left(x_{j+k}-x_{j}\right)^{2}=2, \\
\sum_{k} c_{k}\left(x_{j+k}-x_{j}\right)^{3} & =0, \quad \sum_{k} c_{k}\left(x_{j+k}-x_{j}\right)^{4}=0 . &
\end{array}
$$

Due to the last condition, at least one $c_{k}$ with $k \neq 0$ must be negative.

Example 4.4. The classical fourth order approximation to the second derivative on a periodic domain is given by

$$
h^{2}\left(D_{2} \mathbf{u}\right)_{i}=-\frac{1}{12}\left(\mathbf{u}_{i+2}+\mathbf{u}_{i-2}\right)+\frac{4}{3}\left(\mathbf{u}_{i+1}+\mathbf{u}_{i-1}\right)-\frac{5}{2} \mathbf{u}_{i}
$$

Lemma 4.3 can be used to prove the last main observation of this article.

Theorem 4.5. If $D_{2}$ is a discrete derivative operator approximating the second derivative with order of accuracy $p>2$, it is not dissipative for every entropy.

Proof. Consider the grid point $x_{j}$. Writing the approximation of the second derivative as in (46), there is some coefficient $c_{k}<0, k \neq 0$, due to Lemma 4.3. Fix $\mathbf{u}_{j+k}<0$, say $\mathbf{u}_{j+k}=-1$. Set $\mathbf{u}_{j}=\varepsilon>0$, where $\varepsilon>0$ will be fixed later. Choose $\mathbf{u}_{i}=0$ for $i \neq j, j+k$. Finally, consider the entropy $U(u)=\max \{0, u-\varepsilon / 2\}$. Then, $U^{\prime}(u)=0$ for $u<\varepsilon / 2$ and $U^{\prime}(u)=1$ for $u>\varepsilon / 2$. Thus,

$$
\sum_{i} U^{\prime}\left(\mathbf{u}_{i}\right) \cdot\left(D_{2} \mathbf{u}\right)_{i}=U^{\prime}\left(\mathbf{u}_{j}\right) \cdot\left(D_{2} \mathbf{u}\right)_{j}=1 \cdot \sum_{l} c_{l} \mathbf{u}_{j+l}=c_{0} \underbrace{\mathbf{u}_{j}}_{=\varepsilon}+\underbrace{c_{k}}_{<0} \underbrace{\mathbf{u}_{j+k}}_{<0}>0
$$

if $\varepsilon>0$ is chosen small enough.

Remark 4.6. The entropy $U$ used in the proof of Theorem 4.5 can be made smooth by suitable modifications around $u=\varepsilon / 2$.

Remark 4.7. Of course, higher order approximations to second derivatives that are dissipative for a specific entropy can be constructed. Classical difference operators are negative semidefinite, i.e. they are dissipative for the $L^{2}$ entropy $U(u)=\frac{1}{2} u^{2}$ with $U^{\prime}(u)=u$. For a general entropy $U$, entropy dissipative second derivatives can be constructed by using the entropy variables $w:=U^{\prime}(u)$ instead of the conserved variables $u$, cf. [10].

Remark 4.8. In periodic domains, the classical central finite difference approximations to the first derivative of higher order can be constructed via extrapolation from the second order operator, cf. [31, Section 3.2]. Thus, by enforcing positivity of the corresponding coefficients for the second derivative, entropy dissipative terms can be constructed similarly for higher order first derivative operators, as used in [41]. However, these are not higher order approximations of the second derivative.

\section{Summary and Discussion}

In this article, product and chain rules using averaged compositions have been shown to hold for second order approximations to first order derivative operators, similarly to corresponding results for functions of bounded variation (Lemma 3.1 and Lemma 3.2). While such mimetic properties may have nice implications, it is proven that such results cannot hold for higher order approximations, independently of the grid or the exact form of the discrete derivative operator (Theorem 3.6 and Theorem 3.8). This result holds also for spectral collocation and nodal discontinuous Galerkin methods. 
Furthermore, the entropy dissipation induced by difference operators approximating second derivatives with varying coefficients is studied. While certain second order approximations are dissipative for all entropies (Theorem 4.1), such a result is not valid for higher order approximations (Theorem 4.5).

These results (Theorems 3.6, 3.8, 4.5) have been proven for linear difference operators. Indeed, they rely on Lemma 3.5 and Lemma 4.3, which assume linearity. Thus, similar to classical results for (scalar) conservation laws [19], it might be possible to construct nonlinear operators approximating the first and second derivative such that desirable properties can be obtained.

While these results are interesting on their own, there are several connections with other results and open questions. It is well known that higher order schemes can be more efficient for certain problems than lower order ones [20]. However, the numerical treatment of discontinuities in solutions to hyperbolic conservation laws has to be well-considered, especially for higher order schemes. Even though a single entropy inequality can be sufficient for genuinely nonlinear scalar conservation laws [7, 22, 29], general conservation laws pose additional challenges [23]. Since certain mimetic properties discussed in this article are limited to second order schemes, suitable detection of discontinuities and corresponding adaptations of the numerical methods may be inevitable.

\section{Acknowledgements}

This work was supported by the German Research Foundation (DFG, Deutsche Forschungsgemeinschaft) under Grant SO 363/14-1. The author would like to thank the anonymous reviewers for their helpful comments and valuable suggestions to improve this article.

\section{References}

[1] S. Bianchini and A. Bressan. "Vanishing viscosity solutions of nonlinear hyperbolic systems". In: Annals of Mathematics 161.1 (2005), pp. 223-342. DoI: 10.4007/annals . 2005. 161.223.

[2] M. H. Carpenter, D. Gottlieb, and S. Abarbanel. "Time-Stable Boundary Conditions for Finite-Difference Schemes Solving Hyperbolic Systems: Methodology and Application to High-Order Compact Schemes". In: Journal of Computational Physics 111.2 (1994), pp. 220236. DOI: $10.1006 / \mathrm{jcph} .1994 .1057$.

[3] M. H. Carpenter, J. Nordström, and D. Gottlieb. "A Stable and Conservative Interface Treatment of Arbitrary Spatial Accuracy". In: Journal of Computational Physics 148.2 (1999), pp. 341-365. DOI: 10.1006/jcph. 1998.6114.

[4] T. Chen and C.-W. Shu. "Entropy stable high order discontinuous Galerkin methods with suitable quadrature rules for hyperbolic conservation laws". In: Journal of Computational Physics 345 (2017), pp. 427-461. Dor: 10.1016/j . jcp. 2017.05 .025$.

[5] C. M. Dafermos. Hyperbolic Conservation Laws in Continuum Physics. Berlin Heidelberg: Springer-Verlag, 2010. Dor: 10.1007/978-3-642-04048-1.

[6] G. Dal Maso, P. G. LeFloch, and F. Murat. "Definition and weak stability of nonconservative products". In: Journal de Mathématiques Pures et Appliquées 74.6 (1995), pp. 483548 .

[7] C. De Lellis, F. Otto, and M. Westdickenberg. "Minimal entropy conditions for Burgers equation". In: Quarterly of Applied Mathematics 62.4 (2004), pp. 687-700. Dor: 10 . 1090 / qam/2104269.

[8] L. C. Evans and R. F. Gariepy. Measure Theory and Fine Properties of Functions. Boca Raton: Taylor \& Francis Group, LLC, 2015. 
[9] D. C. D. R. Fernández, J. E. Hicken, and D. W. Zingg. "Review of summation-by-parts operators with simultaneous approximation terms for the numerical solution of partial differential equations". In: Computers \& Fluids 95 (2014), pp. 171-196. DoI: 10 . 1016/j . compfluid.2014.02.016.

[10] T. C. Fisher and M. H. Carpenter. "High-order entropy stable finite difference schemes for nonlinear conservation laws: Finite domains". In: Journal of Computational Physics 252 (2013), pp. 518-557. DoI: 10.1016/j . jcp.2013.06.014.

[11] U. S. Fjordholm, S. Mishra, and E. Tadmor. "Arbitrarily High-Order Accurate Entropy Stable Essentially Nonoscillatory Schemes for Systems of Conservation Laws". In: SIAM Journal on Numerical Analysis 50.2 (2012), pp. 544-573. Dor: 10.1137/110836961.

[12] G. J. Gassner. "A Skew-Symmetric Discontinuous Galerkin Spectral Element Discretization and Its Relation to SBP-SAT Finite Difference Methods". In: SIAM Journal on Scientific Computing 35.3 (2013), A1233-A1253. DOI: 10 . 1137/120890144.

[13] G. J. Gassner, A. R. Winters, and D. A. Kopriva. "A well balanced and entropy conservative discontinuous Galerkin spectral element method for the shallow water equations". In: Applied Mathematics and Computation 272 (2016), pp. 291-308. Dor: 10 . 1016/j . amc . 2015. 07.014.

[14] G. J. Gassner, A. R. Winters, and D. A. Kopriva. “Split Form Nodal Discontinuous Galerkin Schemes with Summation-By-Parts Property for the Compressible Euler Equations". In: Journal of Computational Physics 327 (2016), pp. 39-66. Dor: 10 .1016/j . jcp. 2016.09.013.

[15] B. Gustafsson, H.-O. Kreiss, and J. Oliger. Time-Dependent Problems and Difference Methods. John Wiley \& Sons, 2013.

[16] E. Hairer, C. Lubich, and G. Wanner. "Geometric numerical integration illustrated by the Störmer-Verlet method". In: Acta Numerica (2003), pp. 399-450. Dor: 10 . 1017 / S0962492902000144.

[17] E. Hairer, C. Lubich, and G. Wanner. Geometric Numerical Integration: Structure-Preserving Algorithms for Ordinary Differential Equations. Vol. 31. Springer Series in Computational Mathematics. Berlin Heidelberg: Springer-Verlag, 2006. Dor: 10. 1007/3-540-30666-8.

[18] E. Hairer, S. P. Nørsett, and G. Wanner. Solving Ordinary Differential Equations I: Nonstiff Problems. Vol. 8. Springer Series in Computational Mathematics. Berlin Heidelberg: Springer-Verlag, 2008. Dor: 10.1007/978-3-540-78862-1.

[19] A. Harten. "On the Nonlinearity of Modern Shock-Capturing Schemes". In: Wave Motion: Theory, Modelling, and Computation. Ed. by A. J. Chorin and A. J. Majda. Vol. 7. Mathematical Sciences Research Institute Publications. New York: Springer, 1987, pp. 147-201. Dor: 10.1007/978-1-4613-9583-6_6.

[20] H.-O. Kreiss and J. Oliger. "Comparison of accurate methods for the integration of hyperbolic equations". In: Tellus 24.3 (1972), pp. 199-215. Dor: 10.1111/j .2153-3490. 1972. tbQ1547.x.

[21] H.-O. Kreiss and G. Scherer. "Finite Element and Finite Difference Methods for Hyperbolic Partial Differential Equations". In: Mathematical Aspects of Finite Elements in Partial Differential Equations. Ed. by C. de Boor. New York: Academic Press, 1974, pp. 195-212.

[22] S. G. Krupa and A. F. Vasseur. Single Entropy Condition for Burgers Equation via the Relative Entropy Method. 2017. arXiv: 1709.05610 [math. AP].

[23] P. G. LeFloch. Hyperbolic Systems of Conservation Laws: The Theory of Classical and Nonclassical Shock Waves. Basel: Birkhäuser, 2002. Dor: 10.1007/978-3-0348-8150-0.

[24] P. G. LeFloch, J.-M. Mercier, and C. Rohde. “Fully Discrete, Entropy Conservative Schemes of Arbitrary Order". In: SIAM Journal on Numerical Analysis 40.5 (2002), pp. 1968-1992. DOr: $10.1137 /$ S003614290240069X.

[25] P. G. Lefloch and A. E. Tzavaras. "Representation of weak limits and definition of nonconservative products". In: SIAM journal on mathematical analysis 30.6 (1999), pp. 1309-1342. DOI: $10.1137 /$ SO036141098341794. 
[26] K. Mattsson. "Summation by parts operators for finite difference approximations of second-derivatives with variable coefficients". In: Journal of Scientific Computing 51.3 (2012), pp. 650-682. DOI: 10. 1007/s10915-011-9525-z.

[27] K. Mattsson, M. Svärd, and J. Nordström. "Stable and Accurate Artificial Dissipation". In: Journal of Scientific Computing 21.1 (2004), pp. 57-79. DOI: 10. 1023/B: JOMP . 0000027955. $75872.3 f$.

[28] S. Mishra and M. Svärd. “On stability of numerical schemes via frozen coefficients and the magnetic induction equations". In: BIT Numerical Mathematics 50.1 (2010), pp. 85-108. DOI: $10.1007 / \mathrm{s} 10543-010-0249-5$.

[29] E. Y. Panov. "Uniqueness of the solution of the Cauchy problem for a first order quasilinear equation with one admissible strictly convex entropy". In: Mathematical Notes 55.5 (1994), pp. 517-525. DOI: 10.1007/BF02110380.

[30] H. Ranocha. "Comparison of Some Entropy Conservative Numerical Fluxes for the Euler Equations". In: Journal of Scientific Computing (Dec. 2017). Dor: 10 . 1007/s10915-0170618-1. arXiv: 1701.02264 [math.NA].

[31] H. Ranocha. "Generalised Summation-by-Parts Operators and Entropy Stability of Numerical Methods for Hyperbolic Balance Laws". PhD thesis. TU Braunschweig, 2018.

[32] H. Ranocha. "Generalised Summation-by-Parts Operators and Variable Coefficients". In: Journal of Computational Physics 362 (Feb. 2018), pp. 20-48. Dor: 10.1016/j . jcp . 2018.02 . Q21. arXiv: 1705.10541 [math.NA].

[33] H. Ranocha. "Shallow water equations: Split-form, entropy stable, well-balanced, and positivity preserving numerical methods". In: GEM - International Journal on Geomathematics 8.1 (Apr. 2017), pp. 85-133. Dor: 10.1007/s13137-016-0089-9. arXiv: 1609.08029 [math.NA].

[34] H. Ranocha, J. Glaubitz, P. Öffner, and T. Sonar. "Stability of artificial dissipation and modal filtering for flux reconstruction schemes using summation-by-parts operators". In: Applied Numerical Mathematics 128 (Feb. 2018). See also arXiv: 1606.00995 [math. NA] and arXiv: 1606.01056 [math.NA], pp.1-23. Dor: 10.1016/j . apnum.2018.01.019.

[35] H. Ranocha, P. Öffner, and T. Sonar. "Extended skew-symmetric form for summationby-parts operators and varying Jacobians". In: Journal of Computational Physics 342 (Apr. 2017), pp. 13-28. DoI: 10.1016/j . jcp.2017.04.044. arXiv: 1511.08408 [math.NA].

[36] H. Ranocha, P. Öffner, and T. Sonar. "Summation-by-parts operators for correction procedure via reconstruction". In: Journal of Computational Physics 311 (Apr. 2016), pp. 299-328. DOr: $10.1016 / \mathrm{j}$. jcp. 2016.02.009. arXiv: 1511.02052 [math.NA].

[37] J.-P. Raymond. "A New Definition of Nonconservative Products and Weak Stability Results". In: Bolletino Della Unione Matematica Italiana 10-B.7 (1996), pp. 681-699.

[38] R. E. Showalter. Monotone Operators in Banach Space and Nonlinear Partial Differential Equations. Providence, Rhode Island: American Mathematical Society, 1997.

[39] B. Sjögreen and H. C. Yee. "On Skew-Symmetric Splitting and Entropy Conservation Schemes for the Euler Equations". In: Numerical Mathematics and Advanced Applications 2009: Proceedings of ENUMATH 2009, the 8th European Conference on Numerical Mathematics and Advanced Applications, Uppsala, July 2009. Ed. by G. Kreiss, P. Lötstedt, A. Målqvist, and M. Neytcheva. Berlin, Heidelberg: Springer, 2010, pp. 817-827. Dor: 10 . 1007/978-3642-11795-4_88.

[40] B. Strand. "Summation by Parts for Finite Difference Approximations for $d / d x$ ". In: Journal of Computational Physics 110.1 (1994), pp. 47-67. Dor: 10.1006/jcph. 1994.1005.

[41] M. Svärd and S. Mishra. "Shock Capturing Artificial Dissipation for High-Order Finite Difference Schemes". In: Journal of Scientific Computing 39.3 (2009), pp. 454-484. DoI: 10.1007/s10915-009-9285-1. 
[42] M.Svärd and J. Nordström. "Review of summation-by-parts schemes for initial-boundaryvalue problems". In: Journal of Computational Physics 268 (2014), pp. 17-38. Dor: 10.1016/ j.jcp.2014.02.031.

[43] E. Tadmor. "Entropy stability theory for difference approximations of nonlinear conservation laws and related time-dependent problems". In: Acta Numerica 12 (2003), pp. 451512. DOr: $10.1017 /$ S0962492902000156.

[44] E. Tadmor. "The numerical viscosity of entropy stable schemes for systems of conservation laws. I". In: Mathematics of Computation 49.179 (1987), pp. 91-103. Dor: 10 . 1090/S00255718-1987-0890255-3.

[45] A. I. Vol'pert. "The spaces BV and quasilinear equations". In: Mathematics of the USSRSbornik 2.2 (1967), pp. 225-267. DOI: 10. 1070/SM1967v002n02ABEH002340.

[46] A. I. Vol'pert and S. I. Hudjaev. Analysis in classes of discontinuous functions and equations of mathematical physics. Dodrecht: Martinus Nijhoff Publishers, 1985.

[47] J. von Neumann and R. D. Richtmyer. "A Method for the Numerical Calculation of Hydrodynamic Shocks". In: Journal of Applied Physics 21.3 (1950), pp. 232-237. Dor: 10. 1063/1.1699639.

[48] N. Wintermeyer, A. R. Winters, G. J. Gassner, and D. A. Kopriva. “An entropy stable nodal discontinuous Galerkin method for the two dimensional shallow water equations on unstructured curvilinear meshes with discontinuous bathymetry". In: Journal of Computational Physics 340 (2017), pp. 200-242. Dor: 10.1016/j . jcp.2017.03.036. 